\title{
Charge Ordering in Manganite and Ferrite Systems
}

Shaobo Cheng ${ }^{1}$, Shiqing Deng ${ }^{2}$, Changsong $\mathrm{Xu}^{3}$, Jing Tao ${ }^{1}$ and Yimei Zhu ${ }^{1}$

${ }^{1}$ Brookhaven National Laboratory, Upton, New York, United States, ${ }^{2}$ University of Science and Technology Beijing, Beijing, Beijing, China (People's Republic), ${ }^{3}$ University of Arkansas, Fayetteville, Arkansas, United States

Charge orderings exist ubiquitously in strongly correlated materials and can be widely used in energy related applications, such as supercapacitors, multiferroic materials, etc. As a typical charge ordering system, $\mathrm{LuFe}_{2} \mathrm{O}_{4}$ possesses periodically arranged $\mathrm{Fe}^{2+}$ and $\mathrm{Fe}^{3+}$ ions in the $\mathrm{FeO}$ double layers. The triangle-shaped sublattice of Fe ions leads to the frustrated states for both spin and charge. [1,2] Here, two different types of charge orderings, coexisting in a single crystalline $\mathrm{LuFe}_{2} \mathrm{O}_{4}$ sample, were directly observed (Fig. 1) by taking advantage of the in-situ cooling transmission electron microscopy (TEM). Specifically, vectors of $\mathrm{q}_{1}=(1 / 3,1 / 3,1 / 2)$ and $\mathrm{q}_{2}=(1 / 3,1 / 3,1)$ can be assigned to express these two charge ordering configurations, respectively (Fig. 1(a) and (b)). According to the real-space atomic models for q1 and q2 (Fig. 1(c) and (d)), q1 charge ordering features an antiferroelectric configuration, while q2 has a ferroelectric arrangement. Considering the space symmetry of $\mathrm{LuFe}_{2} \mathrm{O}_{4}$, there exist three equivalent q vectors for both q1 (q1, q1', q1',) and q2 (q2, q2', q2',) leading to a net q1t vector and a q2t vector along $c$ direction as depicted in Fig. 1(e). By selecting $\mathrm{q}_{1}$ and $\mathrm{q}_{2}$ diffraction spots, charge ordering domains of $\mathrm{q}_{1}$ and $\mathrm{q}_{2}$ can be seen in the dark field TEM images in Fig. 1 (f) and (g) at $90 \mathrm{~K}$, respectively, which present the spatial separation. Since the magnetic properties and charge orderings share the same origin (from Fe ions) in the $\mathrm{LuFe}_{2} \mathrm{O}_{4}$ system, the magnetic domains and charge ordering domains should be coupled, which has been manifested by our recent low temperature Lorentz TEM experiments.[3]

$\mathrm{YMn}_{2} \mathrm{O}_{4}$, which is another strongly correlated material with the isomorphic structure, is unstable in nature but can be synthesized by pulsed laser deposition (PLD) method. In the sample where the $\mathrm{YMn}_{2} \mathrm{O}_{4}$ thin film grown on $\alpha-\mathrm{Al}_{2} \mathrm{O}_{3}$ substrate, $\mathrm{MnO}$ double layer was observed to self-assemble at the interface (Fig. 2(a) and (b)). Since $\mathrm{YMn}_{2} \mathrm{O}_{4}$ holds a similar crystal structure with $\mathrm{LuFe}_{2} \mathrm{O}_{4}$, the charge ordering within the $\mathrm{MnO}$ double layer can be expected. The charge ordering state in $\mathrm{MnO}$ double layers with the Type 3 (CO3) configuration can be detected by electron energy-loss spectroscopy (EELS), which manifests quite different characteristics from the one in $\mathrm{LuFe}_{2} \mathrm{O}_{4}$ (shown Fig. 2(g)). Further density functional theory (DFT) calculation results confirm the lowest energy state of $\mathrm{CO} 3$ configuration. [4]

In conclusion, different types of charge orderings have been identified in both manganite and ferrite systems by multiple TEM techniques. Due to the different orbital occupancies of $\mathrm{Fe}$ and $\mathrm{Mn}, \mathrm{LuFe}_{2} \mathrm{O}_{4}$ and $\mathrm{YMn}_{2} \mathrm{O}_{4}$ present different intrinsic charge ordering configurations. The Lorentz TEM studied in ferrite system reveals the fact that the magnetic phase separation is caused by the spatial separation of the charge ordering domain. The charge-spin coupling has thus been established. The novel charge ordering found in manganite is expected to enhance the global ferroelectricity, since the charge ordering ferroelectric polarization and the geometric polarization are aligned. Our results show the rich physical phenomenon related with charge orderings, which provides a new playground for the emergent science $[5]$. 

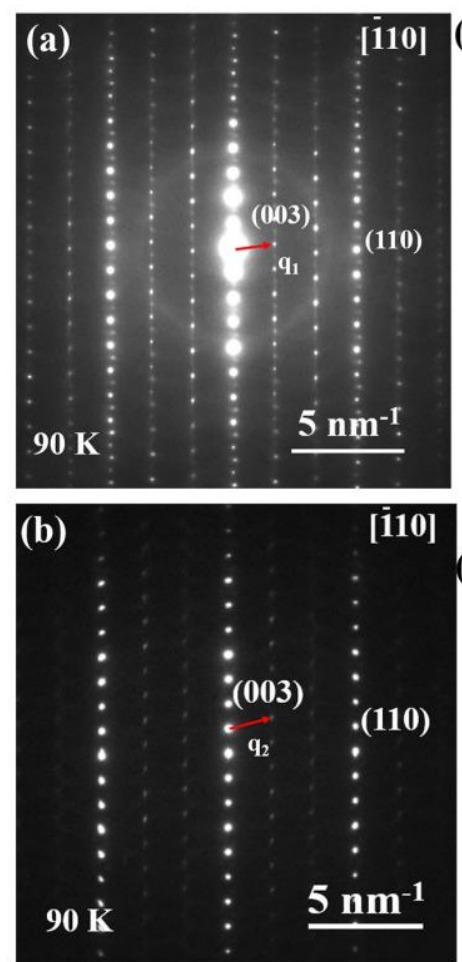

(c) 000000000 ०००००००० ००००००००० 000000000 ०० $\longrightarrow$ ००००० ००००००००० 000000000 000000000 000000000 000000000

(d)

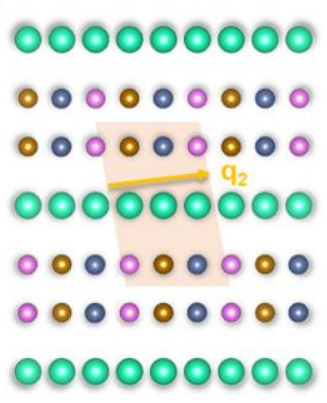

(e)
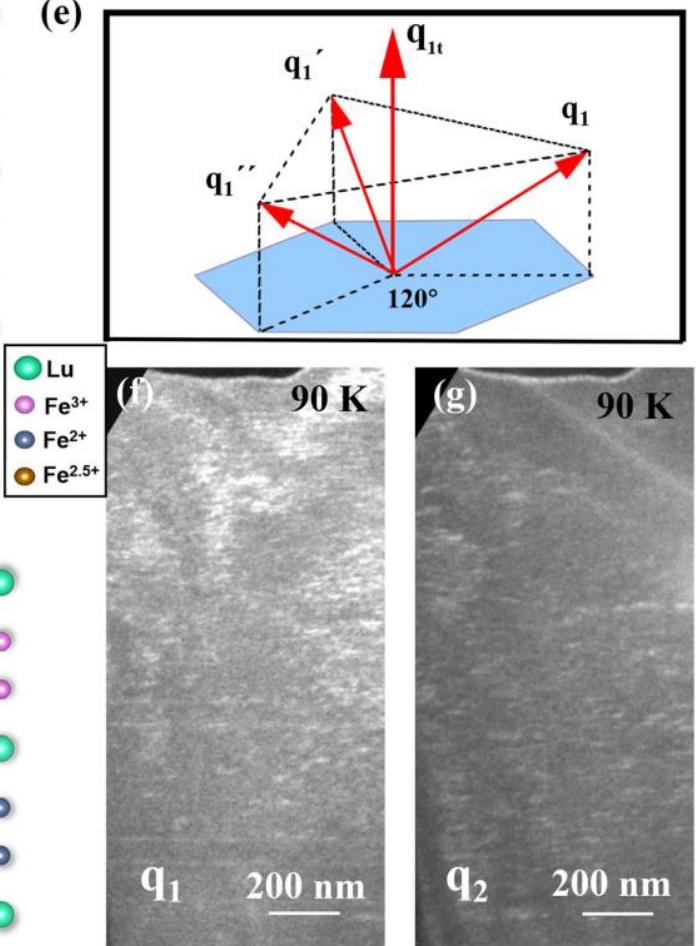

Figure 1. TEM results and atomic models for two different types of charge orderings in LuFe2O4 system. (a) (b) Selected area electron diffraction (SAED) patterns for q1 and q2 charge orderings, respectively, acquired at $90 \mathrm{~K}$ along [-110] direction. (c)(d) The conceived unit cells for the two types of charge orderings. Antiferroelectric configuration for q1, ferroelectric configuration for q2. (e) The resultant qt vector with the consideration of the symmetry of LuFe2O4 unit cell. (f) (g) Low magnification dark field TEM images of the distributions of two types of charge ordering domains of q1 and q2 accordingly.

(a)

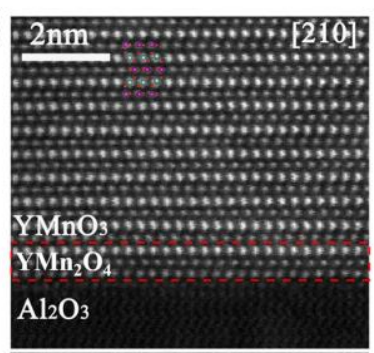

(b)

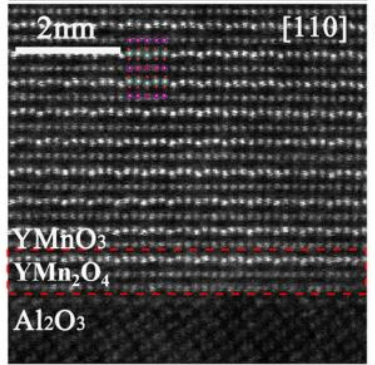

(c) $\mathrm{ST} 1$
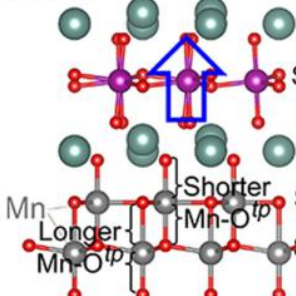

(d)ST2

$\odot$
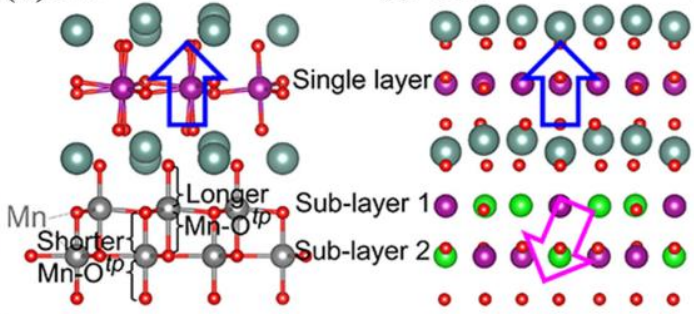

(e) $\mathrm{col}$

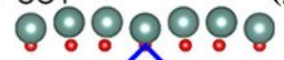

(f) $\mathrm{CO} 2$ (g)cos

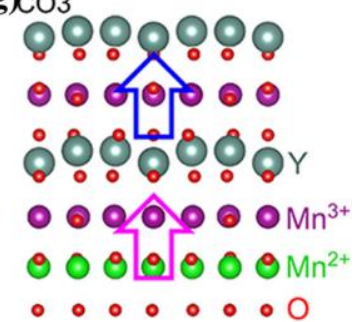

(h) $\mathrm{CO} 4$

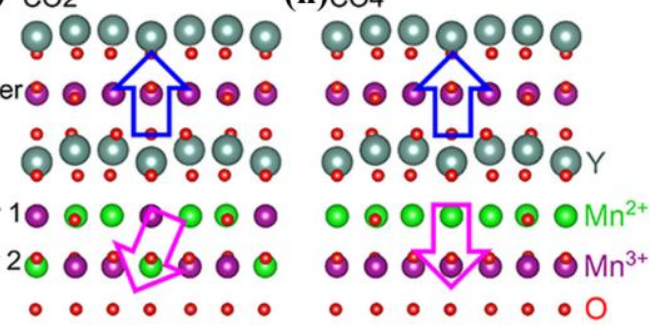

Figure 2. TEM results and atomic models of the charge ordering in manganite systems. (a) HAADFSTEM image showing the interface between $\mathrm{YMnO}_{3}$ film and $\alpha-\mathrm{Al}_{2} \mathrm{O} 3$ substrate along [210] direction. A reconstructed $\mathrm{MnO}$ double layer can be found at the interface, making a nominal $\mathrm{YMn}_{2} \mathrm{O}_{4}$ layer. The 
atomic model of $\mathrm{YMnO}_{3}$ is embedded. (b) HAADF image taken along [110] direction showing the interfacial reconstruction. (c)(d) Two different initial configurations ST1 and ST2 for DFT calculations. ST1 has longer Mn-Otp bonds in sublayer 2 than in sublayer 1, whereas ST2 has the reversed case. (e)-

(h) Four different types of charge orderings in $\mathrm{MnO}$ double layers for DFT calculations. $\mathrm{CO}_{3}$ has the lowest energy among all these four configurations. The blue arrows indicate the geometric ferroelectricity, while the pink arrows indicate the ferroelectricity contributed from charge ordering.

\section{References}

[1] S. Deng, et al,. Physical Review Letters 122 (2019) 126401

[2] M. Angst, et al,. Physical Review Letters 101 (2008) 227601

[3] S. Cheng, et al,. To be submitted

[4] S. Cheng, et al,. Science Advances 4 (2018) eaar4298

[5] Electron microscopy work at BNL was supported by the U.S. Department of Energy, Office of Basic Energy Science, Division of Materials Science and Engineering, under Contract No. DE-SC0012704. This work is supported by the Quantum Materials for Energy Efficient Neuromorphic Computing, an Energy Frontier Research Center funded by the U.S. Department of Energy (DOE), Office of Science, Basic Energy Sciences (BES), under Award DE-SC0019273 